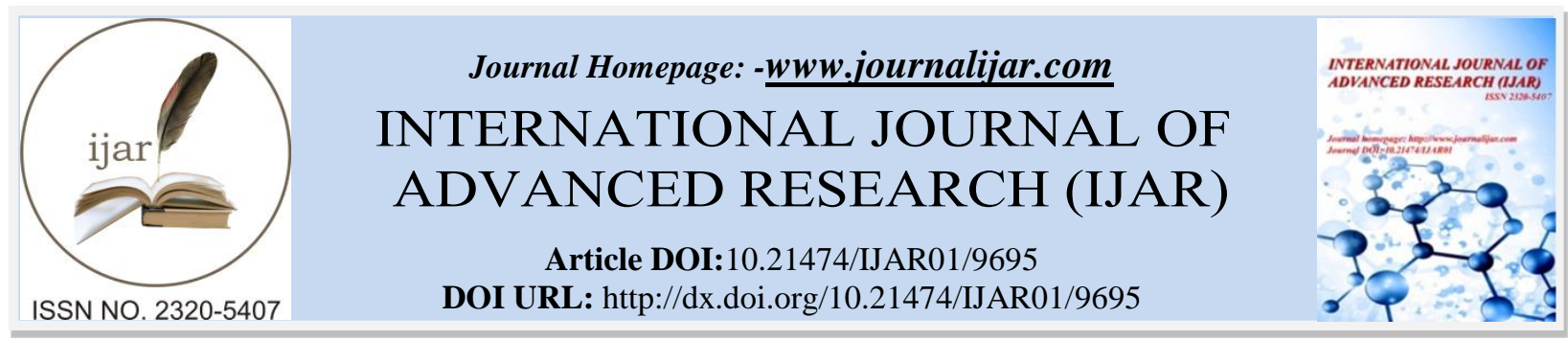

RESEARCH ARTICLE

\title{
LA MUCOPOLYSACCHARIDOSE DE TYPE I: EFFICACITE DU TRAITEMENT ENZYMATIQUE SUBSTITUTIF PAR LA LARONIDASE CHEZ UN CAS.
}

\section{Fadoua Bouzid $^{1}$, Zahra Brakez ${ }^{2}$, Ilham Benyamna ${ }^{1}$, Abdelhamid Elmousadik ${ }^{1}$, Bouchaib Jabir ${ }^{3}$ and Najat} Alif ${ }^{1}$.

1. Laboratoire de Biotechnologies et Valorisation des Ressources Naturelles, département de biologie, université IBN ZOHR, Agadir, Maroc.

2. Laboratoire de Biologie Cellulaire et Génétique Moléculaire département de biologie, université IBN ZOHR, Agadir, Maroc.

3. Cabinet pédiatrique, Agadir, Maroc.

\section{Manuscript Info}

Manuscript History

Received: 08 July 2019

Final Accepted: 10 August 2019

Published: September 2019

Key words:

Mucopolysaccharidosis type I, enzyme replacement therapy, laronidase, storage diseases.

\section{Abstract}

Mucopolusaccharidosis Type I (MPS I) is a lysosomal storage disease due to $\alpha$-L-iduronidase deficiency. Currently, the disease is treated by the recombinant human $\alpha$-L-iduronidase (laronidase). This study evaluates the clinical outcome and quality of life, after two years of traitement by laronidase in a 10 years aged boy, late diagnosed at age 8 with Hurler-Scheie syndrome. The results of the ETS are encouraging. Indeed, this treatment was benefic since it has improved his quality of life; however it have not cure his disease.

Copy Right, IJAR, 2019,. All rights reserved.

\section{Introduction:}

La mucopolysaccharidose de type I (MPS-I) est une maladie autosomique récessive héréditaire rare, due à un déficit en $\alpha$-L-iduronidase (IDUA), qui entraîne l'accumulation lysosomale des deux glycosaminoglycanes (GAGs): le dermatane sulfate et l'héparane sulfate [1,2]. La MPS I présente une grande variabilité phénotypique. Elle se caractérise par des manifestations cliniques multisystémiques, qui progressent depuis la première année de vie.

On distingue trois présentations cliniques [3]:

1. Le syndrome de Hurler;

2. Le syndrome de Hurler-Scheie;

3. Le syndrome de Scheie.

En 2003, la laronidase, ou $\alpha$-L-iduronidase humaine recombinante, commercialisé au Maroc par Sanofi-Aventis sous le nom de, Aldurazyme, a été autorisée comme ETS pour le traitement de la MPS I, sous forme de perfusion intraveineuse hebdomadaire, et à vie [4]. Ce traitement réduit les accumulations anormales des GAGs dans les différents tissus et diminue ainsi leurs excrétions urinaires [5]. Cependant, la ETS ne peut pas franchir la barrière hémato-encéphalique, par conséquent, le système nerveux central n'est pas guéri. L'association de l'ETS et de la greffe de cellules souches hématopoïétiques (GCSH) a fourni le meilleur rapport bénéfice/risque [6].

Dans ce travail, nous rapportons le cas d'un enfant de 10 ans atteint du syndrome de Hurler-Scheie et son évolution après 24 mois de traitement à l'ETS.

CorrespondingAuthor: Fadoua Bouzid.

Address: Laboratoire de Biotechnologies et Valorisation des Ressources Naturelles, département de biologie, université IBN ZOHR, Agadir, Maroc. 


\section{Observation:}

Il s'agit d'une étude conduite, après le consentement éclairé de la famille, au laboratoire de Biotechnologies et Valorisation des Ressources Naturelles (LBVRN) à la faculté de sciences d'Agadir, Cette étude porte sur un patient atteint de la mucopolysaccharidose de type I. Il a été recruté dans le service de pédiatrie de l'hôpital Hassan II d'Agadir. C'est un garçon de dix ans, issu d'un mariage consanguin de premier degré, le troisième enfant de deux frères qui sont phénotypiquement sains et provenant de la région de Chtouka Ait Baha au sud du Maroc. Le patient est diagnostiqué, tardivement, avec un syndrome de Hurler-Scheie à l'âge de 8 ans, Il présente un faciès grossier, une macrocéphalie depuis la naissance et un cou court. Il a une voix de cheval et prononce la lettre S-T. L'examen ophtalmologique a montré une sérieuse opacité de la cornée qui a été constatée à l'âge de 12 mois. L'abdomen était prédominant avec des hernies, inguinale et ombilicale. L'échographie avait montré une hauteur de la rate de 125 $\mathrm{mm}$. Des problèmes respiratoires et des déformations du squelette ont été observés à huit ans. L'examen clinique avait révélé une flexion-extension de l'épaule à environ $100^{\circ}$. Il avait un retard de croissance staturo-pondérale. L'examen radiologique avait montré une dysplasie du bassin avec luxation bilatérale des hanches et une cardiomégalie. Il se fatiguait au moindre effort et on devait le porter, car il ne pouvait pas marcher, même pour une petite distance. Son QI est égal à 100. Devant la suspicion de la maladie de surcharge lysosomale, l'analyse biochimique a montré une forte élévation des GAGs urinaires à $69.6 \mathrm{mg}$ d'acides hexuroniques $/ \mathrm{g}$ de créatinine contre une fourchette normale à son âge de 3,0 à $16,0 \mathrm{mg} / \mathrm{g}$ de créatinine, avec une excrétion importante du dermatane sulfate et de l'héparane sulfate. L'activité enzymatique de l' $\alpha$-L-iduronidase mesurée chez notre patient était nulle. L'ensemble des résultats est évocateur d'une mucopolysaccharidose de type I. L'étude génétique a identifié la mutation p.P533R du gène (IDUA) à l'état homozygote chez cet enfant (Figue 2), qui est considérée comme étant la mutation la plus fréquente chez les patients d'Afrique du Nord [7].

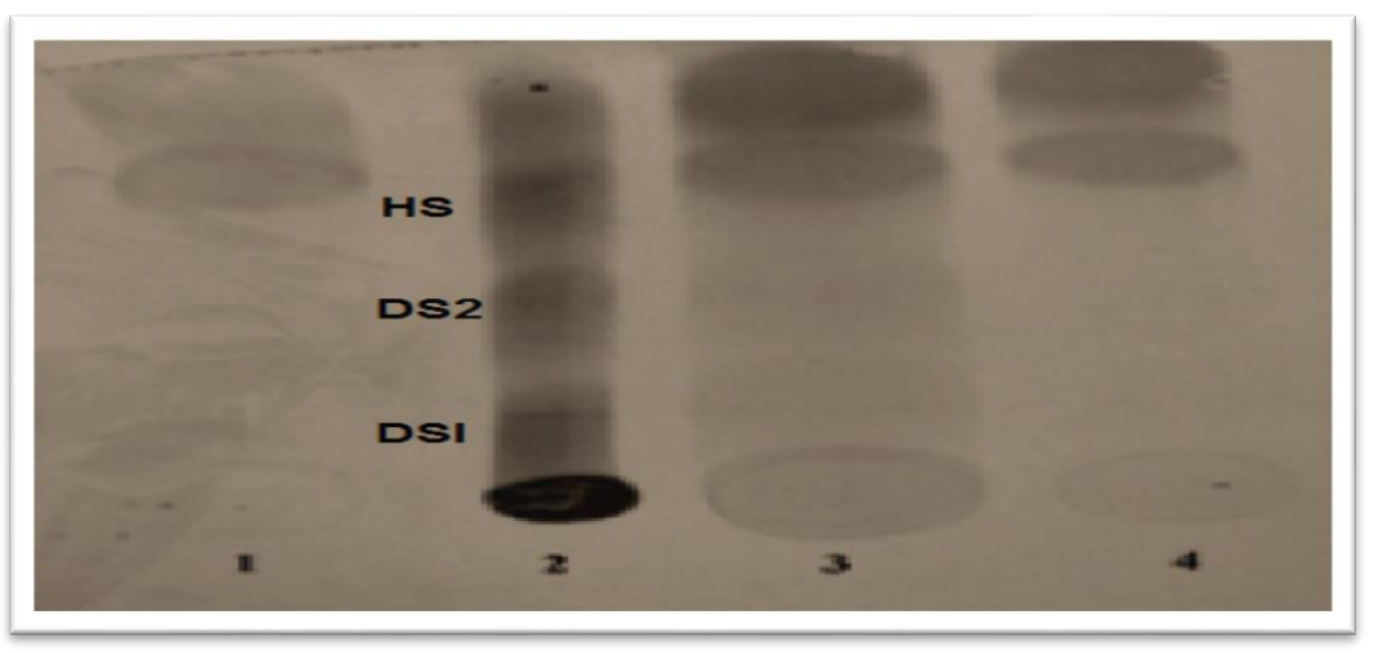

Figure 1: Profil chromatographique des GAGs urinaires sur plaque d'acétate de cellulose. 1: standard de chondroïtine-4-sulfate, 2: Patient Hurler non traité, 3: Patient Hurler-Sheie traité par perfusion de la laronidase et 4: Témoin sain.

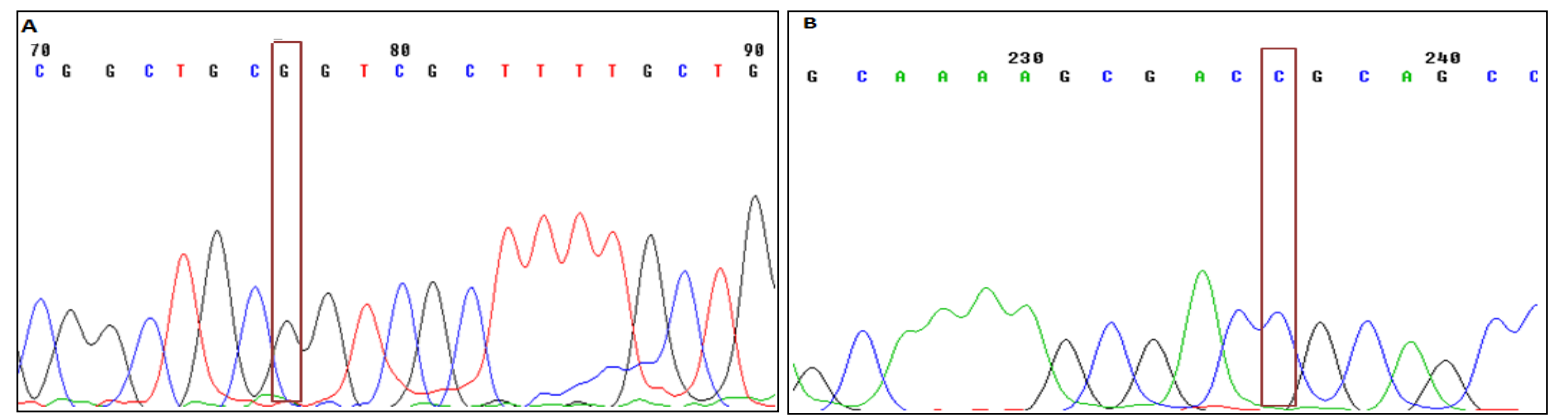

Figure 2:Profil de la séquence au niveau de l'exon XI du gène IDUA montrant la mutation p.Pro $533 \operatorname{Arg}(\mathrm{C}>\mathrm{G})$ chez notre cas. A: Séquence sens; B: Séquence anti-sens. 
Une enzymothérapie substitutive (ETS) par perfusion de la laronidase a été administrée, pendant quatre heures, à la posologie de 100 unités $/ \mathrm{kg}$ par semaine, soit $0,58 \mathrm{mg} / \mathrm{Kg}$. Il avait huit ans quand il a commencé cette thérapie. Le garçon a été traité à l'hôpital Hassan II d'Agadir puis, ultérieurement, dans un centre de santé local à Belfaa, Chtouka Ait Baha. Parmi tous les patients MPS I que nous avons étudié, il est l'unique à avoir bénéficié de l'enzymothérapie. La surveillance de l'efficacité du traitement comprenait un suivi clinique multidisciplinaire et des contrôles en série des GAGs urinaires, effectués tous les trois mois, au cours des deux années.

Avec un recul de 24 mois d'ETS, les valeurs des GAGs ont diminué de $73 \%$ après les trois premiers mois, mais ils sont restés légèrement élevés de $11 \%$ par rapport à la limite supérieure des sujets normaux. Ce résultat a été confirmé par le modèle CCM des GAGs, qui montre qu'ils sont plus accumulés par rapport au contrôle sain, mais moins chez les patients Hurler non traités (figure 1). Ses performances physiques se sont améliorés, il se fatigue moins et joue au foot avec ses congénères. Le test de marche de 6 minutes a été évalué à $440 \mathrm{~m} \pm 10$. La flexion et l'extension des épaules ainsi que l'extension totale des coudes étaient modérément limités à environ $160^{\circ}$, valeur supérieure à celle des patients non traités $\left(<120^{\circ}\right)$ mais toujours inférieure à la valeur de la population en bonne santé $\left(180^{\circ}\right)$. La hauteur de la rate a été réduite de $125 \mathrm{~mm}$ à $110 \mathrm{~mm}$ après 2 ans. La taille du foie a continué d'être normale. Il n'y avait pas d'amélioration des déformations squelettiques, ni de la vitesse de croissance, l'enfant présentait toujours une opacité de la cornée chronique, et il a développé une surdité qui n'avait pas été constatée lors de diagnostic.

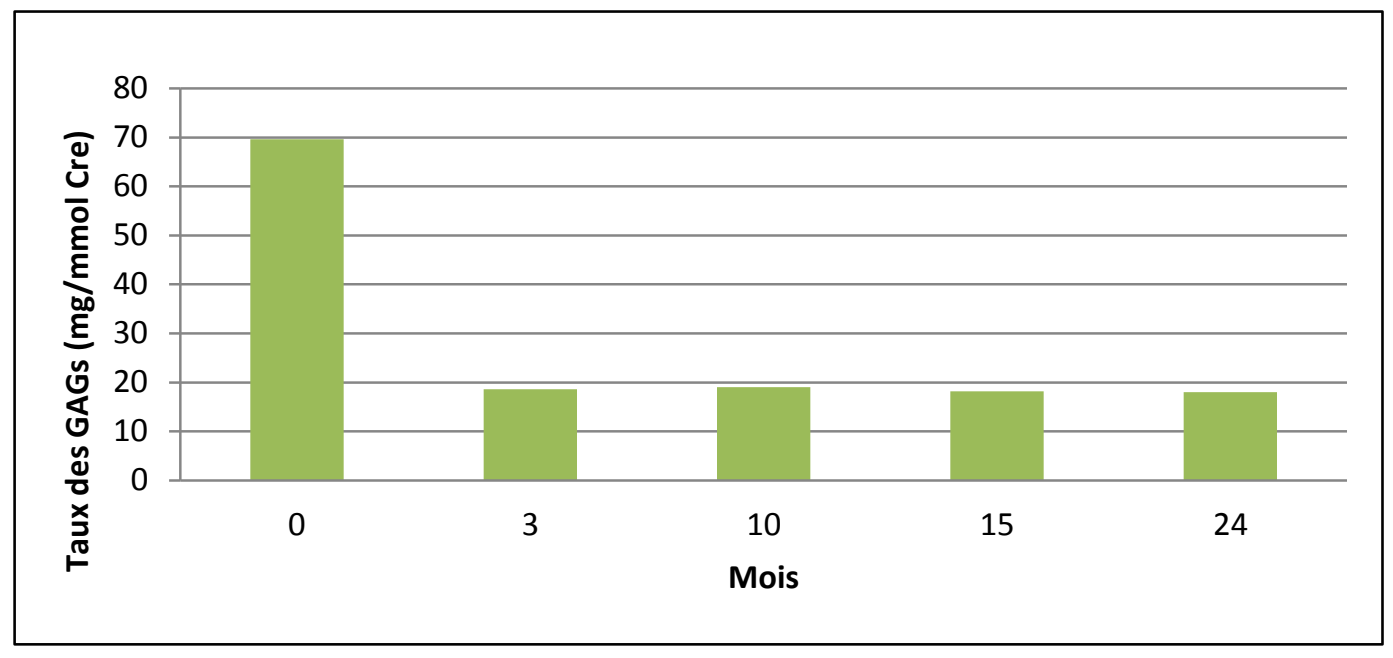

Figure 3: Evolution des GAGs (mg/mmol de créatinine) pendant 24 mois de traitement enzymatique substitutif.

\section{Discussion:}

L'observation présentée est un syndrome de Hurler-Scheie, avec un tableau clinique caractéristique comprenant des traits du visage grossiers, l'opacité cornéenne, l'hépatosplénomégalie, des malformations squelettiques avec des raideurs articulaires et une mobilité réduite.

Après 24 mois du traitement (ETS), les résultats cliniques et l'amélioration de la qualité de vie ont été évalués sur la base des fonctions, pulmonaire et cardiaque, de l'évaluation des déformations articulaires, de la mobilité et de l'ambulation ainsi que du taux des (GAGs) dans l'urine [8].

Dans cette étude, la première preuve de l'efficacité de l'ETS est la réduction du taux des GAGs urinaires. En effet, des diminutions significatives allant jusqu'à $73 \%$, ont été enregistrées après trois mois de traitement. Ceci est en accord avec les résultats précédemment rapportés [9,10] qui ont montré, d'une part, une réduction importante de GAGs chez les patients dont les symptômes ont été fortement améliorés, et d'autre part, une corrélation entre les valeurs des GAGs et l'efficacité de l'ETS. 
Après l'administration de traitement enzymatique substitutif, une amélioration nette a été observée dans l'activité physique exprimée par une tolérance de l'exercice. Nous avons constaté que la distance parcourue dans le test 6 MWT est de $440 \mathrm{~m} \pm 10$. Mais cette distance reste inférieure à la limite de la plage médiane correspondant aux enfants de son âge selon Geiger [455,0 - 692,0] [11] et conformément aux précédentes études [12]. Il ne demande plus à être porté lors d'une marche comme il le faisait avant l'enzymothérapie.

Par ailleurs, le traitement ETS a entraîné une amélioration de la taille de la rate dès la troisième semaine du traitement et une stabilisation de l'atteinte cardiaque alors que la taille du foie a continué d'être normale.

Cependant, nous n'avons observé aucune amélioration des déformations squelettiques ni de l'opacité de la cornée. Ces résultats d'examen clinique confirment l'efficacité et l'innocuité de l'ETS dans le traitement des troubles multisystémiques progressifs [13]. En effet, la proportion majeure d'enzyme recombinante est livrée aux organes viscéraux, tels que le foie, les reins et la rate [14], alors que, une seule petite fraction de l'enzyme recombinante peut atteindre le cartilage osseux et l'œil, ce qui explique pourquoi les améliorations de ces organes sont limitées, même après un traitement de deux ans [15].

Une nette amélioration de la capacité vitale du patient a été remarquée. Ces données concordent avec celles des études antérieures publiées [16] montrant que la grande majorité des patients développent des avantages dès les premiers mois de traitement.

L'une des limites de cette étude réside dans le fait que l'évaluation objective, nécessite un suivi sur plusieurs années, pour quantifier la répercussion réelle sur la qualité et l'espérance de vie. Les bénéfices constatés pendant l'étude doivent se maintenir à long terme (étude après cinq et six ans de traitement) afin d'établir une corrélation avec l'amélioration de la qualité de vie de ce patient $[17,18]$.

Actuellement, les traitements qui ont montré une certaine efficacité dans la MPS I sont: la laronidase seule, la GCSH seule, ou les deux associée, la laronidase pouvant être administrée avant ou après la GCSH, selon le syndrome [6]. D'après les recommandations thérapeutiques de la MPS I, l'ETS est indiquée dans la forme sévère avant l'âge de deux ans, suivie par la GCSH, qui est généralement recommandée pour les patients ayant un syndrome de Hurler, même si le rôle des cellules souches reste controversé. En 2001, Kakkis et al. ont proposé l'ETS seule à vie pour les patients ayant la forme Scheie ou Hurler-Scheie [5]. Le traitement à un âge précoce peut ralentir ou empêcher le développement des symptômes [19], mais il reste inefficace pour prévenir les troubles cognitifs. Notre patient n'a pu bénéficier de l'enzymothérapie qu'à l'âge de 8 ans, âge où sa maladie a été diagnostiquée, pourtant il avait commencé à consulter les médecins depuis l'âge d'un an.

En conclusion, un traitement de deux ans par la laronidase chez notre patient déclaré atteint de syndrome de HurlerScheie s'était montré efficace et inoffensif. Il a ralenti la progression de la maladie et a amélioré certains paramètres. La reconnaissance précoce des symptômes plus subtils, associés à cette maladie, par nos médecins devrait être une priorité afin d'assurer un diagnostic immédiat et une initiation du traitement à un âge précoce ce qui conduira à une forte amélioration de la qualité de vie du patient en évitant l'apparition des symptômes irréversibles.

Les patients MPS I devraient continuer à faire l'objet d'une surveillance étroite, car ces études de suivi nous fourniront des informations supplémentaires sur les avantages de l'ETS précoce.

\section{Remerciements :}

Nous remercions la famille du patient pour toutes les informations qu'elle nous a fournies et le Dr. R.Tantaoui pour les examens cliniques et radiologiques.

\section{Références:}

1. Guffon N. (2003): La mucopolysaccharidose de type I, Présentation clinique et traitement actuel. Méd Thér Pédiatr; 6: 91-7.

2. Neufeld E.F, Muenzer J, Scriver C.R, et al (2001): The Metabolic and Molecular Bases of Inherited Diseases. McGraw-Hill, New York; 3421:52.

3. Muenzer J. (2004): The mucopolysaccharidoses: a heterogeneous group of disorders with variable pediatric presentations. J pediatr; 144: 27-34. 
4. Summary of Product Characteristics (SPC). Aldurazyme $100 \mathrm{U} / \mathrm{ml}$ concentrates for solution for infusion, EMA, 2003.

5. Kakkis ED, Muenzer J, Tiller GE, et al (2001): Enzyme-replacement therapy in mucopolysaccharidoses I. N Engl J Med; 344: 182-8.

6. Cox-Brinkman J, Boelens J.J, Wraith J.E, et al (2006): Haematopoietic cell transplantation (HCT) in combination with enzyme replacement therapy (ERT) in patients with Hurler syndrome. Bone Marrow Transplant; 38:17-21.

7. Chkioua L, Ferchichi S, Khedhiri S et al (2007): La mucopolysaccharidose de type I: stratégie diagnostique en Tunisie. Ann Biol Mol Pratique quotidienne; 65:175-9.

8. Caillaud C. (2006): La mucopolysaccharidose de type I: traitements actuels. Dossier du CNHIM; $1152: 23$.

9. Wraith JE, Clarke LA, Beck M et al (2004): Enzyme replacement therapy for mucopolysaccharidosis I: a randomized, double blinded, placebo-controlled, multinational study of recombinant human alpha-Liduronidase (laronidase). J Pediatr; 144: 581-8.

10. Wraith JE. (2005): The first 5 years of clinical experience with laronidase enzyme replacement therapy for mucopolysaccharidosis I. Expert OpinPharmacother; 6: 489-506.

11. Geiger R, Strasak A, Treml B et al (2007): Six-minute walk test in children and adolescents, J. Pediatr; 150: 395-399.

12. Valayannopoulos V, Wijburg FA. (2011): Therapy for the mucopolysaccharidoses. Rheumatology; 50: 49-59.

13. Kakkis ED, McEntee MF, Schmidtchen A et al (1996): Long-term and high-dose trials of enzyme replacement therapy in the canine model of mucopolysaccharidosis I. Biochem Mol Med; 58: 156-67.

14. Sifuentes M, Doroshow et al (2007): Afollow-up study of MPS I patients treated with laronidase enzyme replacement therapy for 6 years. Mol Gent Me tab; 90(2): 171-80.

15. Clarke L.A, Wraith J.E, Beck M et al (2009): Long-termefficacy and safety of laronidase in the treatment of mucopolysaccharidosis I. Pediatrics; 123: 229-40.

16. Tylki-Szymanska A, Marucha J, Jurecka A, et al (2010): Efficacy of recombinant human alpha-L-iduronidase (laronidase) on restricted range of motion of upper extremities in mucopolysaccharidosis type I patients. J Inherit Metab Dis; 33:151-7.

17. Muenzer J, Fisher A. (2004): Advances in the treatment of mucopolysaccharidosis type I. N Engl J Med; 350: 1932-4. 